PROCEEDINGS OF THE

AMERICAN MATHEMATICAL SOCIETY

Volume 134, Number 3, Pages 805-816

S 0002-9939(05)08127-X

Article electronically published on July 19, 2005

\title{
NON-COMMUTATIVE POSITIVE KERNELS AND THEIR MATRIX EVALUATIONS
}

\author{
DMITRY S. KALYUZHNYĬ-VERBOVETZKII AND VICTOR VINNIKOV
}

(Communicated by Joseph A. Ball)

\begin{abstract}
We show that a formal power series in $2 N$ non-commuting indeterminates is a positive non-commutative kernel if and only if the kernel on $N$-tuples of matrices of any size obtained from this series by matrix substitution is positive. We present two versions of this result related to different classes of matrix substitutions. In the general case we consider substitutions of jointly nilpotent $N$-tuples of matrices, and thus the question of convergence does not arise. In the "convergent" case we consider substitutions of $N$-tuples of matrices from a neighborhood of zero where the series converges. Moreover, in the first case the result can be improved: the positivity of a non-commutative kernel is guaranteed by the positivity of its values on the diagonal, i.e., on pairs of coinciding jointly nilpotent $N$-tuples of matrices. In particular this yields an analogue of a recent result of Helton on non-commutative sums-of-squares representations for the class of hereditary non-commutative polynomials. We show by an example that the improved formulation does not apply in the "convergent" case.
\end{abstract}

\section{InTRODUCTION AND STATEMENT OF THE RESUltS}

Several recent results show a relationship between properties of a polynomial or of a formal power series in say $N$ non-commuting indeterminates and the properties of the matrix-valued functions on $N$-tuples of matrices of all sizes obtained from this polynomial or this formal power series by matrix substitution; e.g., see [6, 8, 7, 4, 2].

The objective of this paper is to establish a result of this type in the framework of positive kernels [3, 9, 1. We recall that if $\Omega$ is any set and $\mathcal{E}$ is a Hilbert space, an operator-valued function $K: \Omega \times \Omega \rightarrow \mathcal{L}(\mathcal{E})^{1}$ is called a positive $\mathcal{L}(\mathcal{E})$-valued kernel on $\Omega$ if for any integer $l$, any points $\omega^{(1)}, \ldots, \omega^{(l)} \in \Omega$ and any vectors $h^{(1)}, \ldots, h^{(l)} \in \mathcal{E}$, one has

$$
\sum_{j, k=1}^{l}\left\langle K\left(\omega^{(j)}, \omega^{(k)}\right) h^{(k)}, h^{(j)}\right\rangle_{\mathcal{E}} \geq 0 .
$$

Received by the editors June 13, 2004 and, in revised form, October 19, 2004.

2000 Mathematics Subject Classification. Primary 30C45, 47A56; Secondary 13F25, 47A60.

Key words and phrases. Formal power series, non-commuting indeterminates, positive noncommutative kernels, matrix substitutions, hereditary polynomials, factorization.

The first author was supported by the Center for Advanced Studies in Mathematics, BenGurion University of the Negev.

The second author was partially supported by the Israel Science Foundation Grant 322/00-1.

${ }^{1} \mathcal{L}(\mathcal{E})$ denotes the $C^{*}$-algebra of bounded linear operators on $\mathcal{E}$.

(C)2005 American Mathematical Society Reverts to public domain 28 years from publication 
Alternatively, there exists an auxilliary Hilbert space $\mathcal{H}$ and an operator-valued function $H: \Omega \rightarrow \mathcal{L}(\mathcal{H}, \mathcal{E})^{2}$ so that the factorization

$$
K\left(\omega, \omega^{\prime}\right)=H(\omega) H\left(\omega^{\prime}\right)^{*}
$$

holds for all $\omega, \omega^{\prime} \in \Omega$.

Let $\mathcal{F}_{N}$ denote the free semigroup with $N$ generators $g_{1}, \ldots, g_{N}$ and unit element $\emptyset$. For $w=g_{j_{1}} \cdots g_{j_{m}}$ and $w^{\prime}=g_{k_{1}} \cdots g_{k_{l}}$ from $\mathcal{F}_{N}$ (words in the alphabet $\left.\left\{g_{1}, \ldots, g_{N}\right\}\right)$, the semigroup multiplication is the concatenation

$$
w w^{\prime}=g_{j_{1}} \cdots g_{j_{m}} g_{k_{1}} \cdots g_{k_{l}},
$$

and $\emptyset \in \mathcal{F}_{N}$ plays a role of the empty word: for any $w \in \mathcal{F}_{N}$,

$$
\emptyset w=w \emptyset=w .
$$

For a word $w=g_{j_{1}} \cdots g_{j_{m}} \in \mathcal{F}_{N}$ define its transpose $w^{\top}=g_{j_{m}} \cdots g_{j_{1}} \in \mathcal{F}_{N}$ and its length $|w|=m \in \mathbb{N}$, and define $\emptyset^{\top}=\emptyset,|\emptyset|=0$.

Let $z_{1}, \ldots, z_{d}$ be non-commuting indeterminates, and let $w=g_{j_{1}} \cdots g_{j_{m}} \in \mathcal{F}_{d}$. Set

$$
z^{w}=z_{j_{1}} \cdots z_{j_{m}}, \quad z^{\emptyset}=1 .
$$

For any vector space $\mathcal{L}$ we denote by $\mathcal{L}\left\langle\left\langle z_{1}, \ldots, z_{d}\right\rangle\right\rangle$ the space of all formal power series in $z_{1}, \ldots, z_{d}$ with coefficients in $\mathcal{L}$.

A formal power series in $2 N$ non-commuting indeterminates $z=\left(z_{1}, \ldots, z_{N}\right)$ and $z^{\prime}=\left(z_{1}^{\prime}, \ldots, z_{N}^{\prime}\right)$,

$$
K\left(z, z^{\prime}\right)=\sum_{w, w^{\prime} \in \mathcal{F}_{N}} K_{w, w^{\prime}} z^{w} z^{\prime w^{\prime \top}} \in \mathcal{L}(\mathcal{E})\left\langle\left\langle z, z^{\prime}\right\rangle\right\rangle,
$$

is called a positive non-commutative kernel if $\left(w, w^{\prime}\right) \mapsto K_{w, w^{\prime}}$ is a positive $\mathcal{L}(\mathcal{E})$ valued kernel on $\mathcal{F}_{N}$, i.e., for any integer $l$, any words $w^{(1)}, \ldots, w^{(l)} \in \mathcal{F}_{N}$ and any vectors $h^{(1)}, \ldots, h^{(l)} \in \mathcal{E}$, one has

$$
\sum_{j, k=1}^{l}\left\langle K_{w^{(j)}, w^{(k)}} h^{(k)}, h^{(j)}\right\rangle_{\mathcal{E}} \geq 0 .
$$

Alternatively, there is an auxiliary Hilbert space $\mathcal{H}$ and a non-commutative formal power series

$$
H(z)=\sum_{w \in \mathcal{F}_{N}} H_{w} z^{w} \in \mathcal{L}(\mathcal{H}, \mathcal{E})\langle\langle z\rangle\rangle
$$

such that

$$
K\left(z, z^{\prime}\right)=H(z) H\left(z^{\prime}\right)^{*}
$$

where we use the convention

$$
\left(z^{\prime w}\right)^{*}=z^{\prime w^{\top}} .
$$

This notion of positivity was studied extensively in $[5,3$

\footnotetext{
${ }^{2} \mathcal{L}(\mathcal{H}, \mathcal{E})$ denotes the Banach space of bounded linear operators from $\mathcal{H}$ to $\mathcal{E}$.

3 In [5], it is assumed that $z_{i}$ commutes with $z_{j}^{\prime}$. For our purposes it is more convenient to deal with $2 N$ non-commuting indeterminates; notice that it does not really matter since in all our formulae every $z_{j}^{\prime}$ appears to the right of every $z_{i}$, in every monomial.
} 
Let $Z=\left(Z_{1}, \ldots, Z_{N}\right) \in\left(\mathbb{C}^{n \times n}\right)^{N}$, i.e., $Z$ is an $N$-tuple of $n \times n$ matrices with complex entries, and $w=g_{j_{1}} \cdots g_{j_{m}} \in \mathcal{F}_{N}$. Set

$$
Z^{w}=Z_{j_{1}} \cdots Z_{j_{m}}, \quad Z^{\emptyset}=I_{n} .
$$

Let $n, r \in \mathbb{N}$. Define the set $\operatorname{Nilp}_{N}(n, r)$ of $N$-tuples $Z$ of $n \times n$ matrices with complex entries, which are jointly nilpotent of rank at most $r$, i.e., $Z^{w}=0$ for all $w \in \mathcal{F}_{N}$ such that $|w| \geq r$. Also define the set $\operatorname{Nilp}_{N}(n)=\bigcup_{r=1}^{\infty} \operatorname{Nilp}_{N}(n, r)$ of jointly nilpotent $N$-tuples of $n \times n$ matrices 4

Theorem 1. A formal power series $K\left(z, z^{\prime}\right) \in \mathcal{L}(\mathcal{E})\left\langle\left\langle z, z^{\prime}\right\rangle\right\rangle$ as in (11) is a positive non-commutative kernel if and only if

$$
K\left(Z, Z^{\prime}\right):=\sum_{w, w^{\prime} \in \mathcal{F}_{N}} K_{w, w^{\prime}} \otimes Z^{w} Z^{\prime^{w^{\prime \top}}} \in \mathcal{L}\left(\mathcal{E} \otimes \mathbb{C}^{n}\right),
$$

where $Z, Z^{\prime} \in \operatorname{Nilp}_{N}(n), Z^{\prime *}=\left(Z_{1}^{\prime *}, \ldots, Z_{N}^{\prime *}\right)$ (notice that the sum is therefore finite!), is a positive $\mathcal{L}\left(\mathcal{E} \otimes \mathbb{C}^{n}\right)$-valued kernel on $\operatorname{Nilp}_{N}(n)$ for every $n \in \mathbb{N}$, i.e., for any integer $l$, any jointly nilpotent $N$-tuples of matrices $Z^{(1)}, \ldots, Z^{(l)} \in \operatorname{Nilp}_{N}(n)$ and any vectors $x^{(1)}, \ldots, x^{(l)} \in \mathcal{E} \otimes \mathbb{C}^{n}$, one has

$$
\sum_{j, k=1}^{l}\left\langle K\left(Z^{(j)}, Z^{(k)}\right) x^{(k)}, x^{(j)}\right\rangle_{\mathcal{E} \otimes \mathbb{C}^{n}} \geq 0 .
$$

One direction of the theorem is obvious. If $K\left(z, z^{\prime}\right)$ is a positive non-commutative kernel, then we have a factorization $K\left(z, z^{\prime}\right)=H(z) H\left(z^{\prime}\right)^{*}$ for some formal power series $H(z)$ as in (2) and some Hilbert space $\mathcal{H}$. Therefore for any $Z, Z^{\prime} \in \operatorname{Nilp}_{N}(n)$ we have $K\left(Z, Z^{\prime}\right)=H(Z) H\left(Z^{\prime}\right)^{*}$, where

$$
H(Z):=\sum_{w \in \mathcal{F}_{N}} H_{w} \otimes Z^{w} \in \mathcal{L}\left(\mathcal{H} \otimes \mathbb{C}^{n}, \mathcal{E} \otimes \mathbb{C}^{n}\right)
$$

(notice that the sum is finite). Therefore for each $n \in \mathbb{N}$ we have that $K\left(Z, Z^{\prime}\right)$ is a positive $\mathcal{L}\left(\mathcal{E} \otimes \mathbb{C}^{n}\right)$-valued kernel on $\operatorname{Nilp}_{N}(n)$.

Section 2 contains the proof of the other direction of the theorem.

Note that by using nilpotent matrix substitutions one avoids any convergence assumptions on the formal power series. There is also a "convergent" version of Theorem 1. A formal power series $K\left(z, z^{\prime}\right) \in \mathcal{L}(\mathcal{E})\left\langle\left\langle z, z^{\prime}\right\rangle\right\rangle$ as in (11) is called convergent if for every $n \in \mathbb{N}$ there exists a connected open neighbourhood $U_{n}$ of 0 in $\left(\mathbb{C}^{n \times n}\right)^{N}$ such that the series $K\left(Z, Z^{\prime}\right)$ as in (3) converges uniformly on compact subsets of $U_{n} \times U_{n}$ in the norm of $\mathcal{L}\left(\mathcal{E} \otimes \mathbb{C}^{n}\right)$ (we assume that $\mathcal{F}_{N}$ and $\mathcal{F}_{2 N} \cong \mathcal{F}_{N} \times \mathcal{F}_{N}$ are ordered, say, lexicographically).

Theorem 2. A convergent formal power series $K\left(z, z^{\prime}\right) \in \mathcal{L}(\mathcal{E})\left\langle\left\langle z, z^{\prime}\right\rangle\right\rangle$ (with respect to a set of neighbourhoods $U_{n}$ of 0 in $\left.\left(\mathbb{C}^{n \times n}\right)^{N}, n \in \mathbb{N}\right)$ is a positive noncommutative kernel if and only if $K\left(Z, Z^{\prime}\right)$ is a positive $\mathcal{L}\left(\mathcal{E} \otimes \mathbb{C}^{n}\right)$-valued kernel on $U_{n}$ for every $n \in \mathbb{N}$.

We prove this theorem in Section 3.

In the "nilpotent" case there is a considerable strengthening of Theorem 1. The authors thank the anonymous referee for suggesting this result and its proof.

\footnotetext{
${ }^{4}$ Let us remark that a jointly nilpotent tuple of matrices is jointly similar to a tuple of strictly upper triangular matrices - the authors thank Leonid Gurvits for pointing this out.
} 
Theorem 3. A formal power series $K\left(z, z^{\prime}\right) \in \mathcal{L}(\mathcal{E})\left\langle\left\langle z, z^{\prime}\right\rangle\right\rangle$ as in (11) is a positive non-commutative kernel if and only if for every $n \in \mathbb{N}$ and $Z \in \operatorname{Nilp}_{N}(n)$,

$$
K(Z, Z):=\sum_{w, w^{\prime} \in \mathcal{F}_{N}} K_{w, w^{\prime}} \otimes Z^{w} Z^{* w^{\prime \top}} \in \mathcal{L}\left(\mathcal{E} \otimes \mathbb{C}^{n}\right)
$$

is a positive semidefinite operator, i.e., for any $x \in \mathcal{E} \otimes \mathbb{C}^{n}$ one has

$$
\langle K(Z, Z) x, x\rangle_{\mathcal{E} \otimes \mathbb{C}^{n}} \geq 0 .
$$

One direction of the theorem is obvious due to the remark following the formulation of Theorem 1 and the fact that the operator $H(Z) H(Z)^{*}$ is positive semidefinite. Section 4 contains the proof of the other direction of Theorem 3. While Theorem 3 implies Theorem 11. Section 2 contains an independent proof of Theorem 1 since the argument of this proof is also used in the proof of Theorem 2 , Let us also remark that the statement analogous to the one of Theorem 3 does not hold in the "convergent" case. Indeed, set $K\left(z, z^{\prime}\right):=1-z z^{\prime} \in \mathbb{C}\langle z\rangle$ (here $N=1$ ). Clearly, $K(Z, Z)>0$ for a matrix $Z$ (of any size) close to the origin, however $K\left(z, z^{\prime}\right)$ is not a positive kernel since its matrix of coefficients $\left(\begin{array}{cc}1 & 0 \\ 0 & -1\end{array}\right)$ is indefinite.

Theorem 3 implies a factorization result for a class of non-commutative polynomials. For a vector space $\mathcal{L}$ we denote by $\mathcal{L}\left\langle z_{1}, \ldots, z_{d}\right\rangle$ the space of all polynomials in non-commuting indeterminates $z_{1}, \ldots, z_{d}$ with coefficients in $\mathcal{L}$, i.e., a subspace in $\mathcal{L}\left\langle\left\langle z_{1}, \ldots, z_{d}\right\rangle\right\rangle$ consisting of a formal power series with a finitely supported set of coefficients. An operator-valued polynomial in $2 N$ non-commuting indeterminates $z=\left(z_{1}, \ldots, z_{N}\right)$ and $z^{\prime}=\left(z_{1}^{\prime}, \ldots, z_{N}^{\prime}\right)$ is called called hereditary if it is of the form

$$
K\left(z, z^{\prime}\right)=\sum_{w, w^{\prime} \in \mathcal{F}_{N}:|w| \leq m,\left|w^{\prime}\right| \leq m} K_{w, w^{\prime}} z^{w} z^{\prime w^{\prime T}} \in \mathcal{L}(\mathcal{E})\left\langle z, z^{\prime}\right\rangle,
$$

where $\mathcal{E}$ is a Hilbert space, i.e., every $z_{j}^{\prime}$ appears to the right of every $z_{i}$, in every monomial.

Theorem 4. A hereditary polynomial $K\left(z, z^{\prime}\right) \in \mathcal{L}(\mathcal{E})\left\langle z, z^{\prime}\right\rangle$ as in (6) satisfies $K(Z, Z) \geq 0$ for all $Z \in \operatorname{Nilp}_{N}(n)(n=1,2, \ldots)$ if and only if there exist an auxiliary Hilbert space $\mathcal{H}$ (of dimension at most $\operatorname{dim}(\mathcal{E}) \sum_{j=0}^{m} N^{j}$ ) and a polynomial

$$
H(z)=\sum_{w \in \mathcal{F}_{N}:|w| \leq m} H_{w} z^{w} \in \mathcal{L}(\mathcal{H}, \mathcal{E})\langle z\rangle
$$

such that

$$
K\left(z, z^{\prime}\right)=H(z) H\left(z^{\prime}\right)^{*} .
$$

Furthermore, for this factorization to hold it is enough to assume that $K(Z, Z) \geq 0$ holds for all $Z \in \operatorname{Nilp}_{N}\left(\sum_{j=0}^{m} N^{j}, m+1\right)$.

We deduce this theorem in Section 4. Theorem 4 is an analogue of the main result of [6] (for related results and generalizations see [8] and [7]), for hereditary non-commutative polynomials and jointly nilpotent test matrices. 
In view of the results of this paper it is of interest to investigate the relationship between the non-commutative formal reproducing kernel Hilbert space associated to a positive non-commutative kernel (such spaces are studied in [5]) and the reproducing kernel Hilbert spaces associated to the kernels obtained from the non-commutative kernel by matrix substitutions.

\section{Proof of Theorem 1}

For the proof of Theorem 1 we need the following lemma.

Lemma 1. Let $\mathcal{U}$ be a Hilbert space and let an $\mathcal{L}(\mathcal{U})$-valued polynomial function

$$
P\left(\lambda, \lambda^{\prime}\right)=\sum_{t, t^{\prime} \in \mathbb{Z}_{+}^{N}: 0 \leq|t|,\left|t^{\prime}\right| \leq m} P_{t, t^{\prime}} \lambda^{t}\left(\overline{\lambda^{\prime}}\right)^{t^{\prime}}
$$

be a positive kernel on $\mathbb{C}^{N}$. Here for $\lambda=\left(\lambda_{1}, \ldots, \lambda_{N}\right) \in \mathbb{C}^{N}$ and $t=\left(t_{1}, \ldots, t_{N}\right) \in$ $\mathbb{Z}_{+}^{N}$ we set $\lambda^{t}=\lambda_{1}{ }^{t_{1}} \cdots \lambda_{N}^{t_{N}}$ and $|t|=t_{1}+\cdots+t_{N}$. Then the matrix $M_{P}=$ $\left(P_{t, t^{\prime}}\right)_{0 \leq|t|,\left|t^{\prime}\right| \leq m}$ (for a certain order on $\mathbb{Z}_{+}^{N}$ ) defines a positive semidefinite operator on $\mathcal{U}^{\frac{(m+N) !}{m ! N !} \text {. }}$.

This also holds for sesquianalytic rather than polynomial kernels (of course in this case one has to consider the matrices $\left(P_{t, t^{\prime}}\right)_{0 \leq|t|,\left|t^{\prime}\right| \leq m}$ for any integer $m$ ) and the converse is true as well; note that these facts are a "commutative convergent" analogue of Theorem 1] They are well known for $N=1$ (see, e.g., [1, Lemma 1.1.5]) and the proofs in the general case are analogous. We provide a proof of the lemma for the sake of completeness.

Proof of Lemma 1. For an arbitrary $u=\left(u_{t}\right)_{0 \leq|t| \leq m} \in \mathcal{U}^{\frac{(m+N) !}{m ! N !}}$ we have

$$
\begin{aligned}
& \left\langle M_{P} u, u\right\rangle_{\mathcal{U}}^{\frac{(m+N) !}{m ! N !}}=\sum_{t, t^{\prime} \in \mathbb{Z}_{+}^{N}: 0 \leq|t|,\left|t^{\prime}\right| \leq m}\left\langle P_{t, t^{\prime}} u_{t^{\prime}}, u_{t}\right\rangle_{\mathcal{U}} \\
& =\sum_{t, t^{\prime} \in \mathbb{Z}_{+}^{N}:} \int_{0 \leq|t|,\left|t^{\prime}\right| \leq m}\left\langle P\left(\lambda, \lambda_{\mathbb{T}^{N} \times \mathbb{T}^{N}}\right) u_{t^{\prime}}, u_{t}\right\rangle_{\mathcal{U}} \bar{\lambda}^{t} \lambda^{\prime t^{\prime}} d m_{N}(\lambda) d m_{N}\left(\lambda^{\prime}\right),
\end{aligned}
$$

where $d m_{N}(\cdot)$ is the normalized Lebesgue measure on $\mathbb{T}^{N}$. For an arbitrary $n \in \mathbb{N}$, let $\left\{\Delta_{j}\right\}_{j=1}^{n}$ be a partition of $\mathbb{T}^{N}$ such that $m_{N}\left(\Delta_{j}\right)=\frac{1}{n}$ and let $\lambda^{(j)} \in \Delta_{j}$, $j=1, \ldots, n$, be arbitrary points. Then

$$
\begin{aligned}
& \left\langle M_{P} u, u\right\rangle_{\mathcal{U}} \frac{(m+N) !}{m ! N !} \\
& =\sum_{0 \leq|t|,\left|t^{\prime}\right| \leq m} \lim _{n \rightarrow \infty} \frac{1}{n^{2}} \sum_{j, k=1}^{n}\left\langle P\left(\lambda^{(j)}, \lambda^{(k)}\right) u_{t^{\prime}}, u_{t}\right\rangle_{\mathcal{U}}\left(\overline{\lambda^{(j)}}\right)^{t}\left(\lambda^{(k)}\right)^{t^{\prime}} \\
& =\lim _{n \rightarrow \infty} \frac{1}{n^{2}} \sum_{j, k=1}^{n}\left\langle P\left(\lambda^{(j)}, \lambda^{(k)}\right) \sum_{0 \leq\left|t^{\prime}\right| \leq m}\left(\lambda^{(k)}\right)^{t^{\prime}} u_{t^{\prime}}, \sum_{0 \leq|t| \leq m}\left(\lambda^{(j)}\right)^{t} u_{t}\right\rangle_{\mathcal{U}} \geq 0,
\end{aligned}
$$

as desired.

Proof of Theorem 1. As we already said in Section 1, one direction of this theorem is obvious, and thus only the other one is left to prove. Assume that a formal power series $K\left(z, z^{\prime}\right) \in \mathcal{L}(\mathcal{E})\left\langle\left\langle z, z^{\prime}\right\rangle\right\rangle$ as in (1) is such that the function $K\left(Z, Z^{\prime}\right)$ as in (3) is a positive $\mathcal{L}\left(\mathcal{E} \otimes \mathbb{C}^{n}\right)$-valued kernel on $\operatorname{Nilp}_{N}(n)$ for every $n \in \mathbb{N}$. 
Let $m \in \mathbb{N}, m>1$. Set $n=(N+1)^{m}$. Define a linear operator $S$ on the space $\mathbb{C}^{n} \cong\left(\mathbb{C}^{N+1}\right)^{\otimes m}$ by its action on the basis vectors $e_{i_{1}} \otimes \cdots \otimes e_{i_{m}}$, where $e_{j}$, $j=1, \ldots, N+1$, are the standard basis vectors in $\mathbb{C}^{N+1}$ :

$$
S\left(e_{i_{1}} \otimes \cdots \otimes e_{i_{m}}\right)=e_{i_{m}} \otimes e_{i_{1}} \otimes \cdots \otimes e_{i_{m-1}} .
$$

The operator $S$ is represented in the basis $e_{i_{1}} \otimes \cdots \otimes e_{i_{m}}, i_{1}, \ldots, i_{m} \in\{1, \ldots, N+1\}$, by a $n \times n$ permutation matrix. Let $E_{i, j} \in \mathbb{C}^{(N+1) \times(N+1)}, i, j=1, \ldots, N+1$, be matrices of the form

$$
\left(E_{i, j}\right)_{\mu, \nu}= \begin{cases}1, & (\mu, \nu)=(i, j) \\ 0, & (\mu, \nu) \neq(i, j)\end{cases}
$$

Define

$$
Z_{k}=\left(E_{k+1,1} \otimes I_{N+1}^{\otimes m-1}\right) S \in \mathbb{C}^{n \times n} \cong\left(\mathbb{C}^{(N+1) \times(N+1)}\right)^{\otimes m}, k=1, \ldots, N .
$$

For $\lambda=\left(\lambda_{1}, \ldots, \lambda_{N}\right) \in \mathbb{C}^{N}$, set

$$
Z(\lambda)=\left(\lambda_{1} Z_{1}, \ldots, \lambda_{N} Z_{N}\right) \in\left(\mathbb{C}^{n \times n}\right)^{N} .
$$

For $w=g_{j_{1}} \cdots g_{j_{|w|}} \in \mathcal{F}_{N}: 0<|w| \leq m$, one has

$$
Z(\lambda)^{w}=\lambda_{j_{1}} Z_{j_{1}} \cdots \lambda_{j_{|w|}} Z_{j_{|w|}}=\lambda^{w}\left(E_{j_{1}+1,1} \otimes \cdots \otimes E_{j_{|w|}+1,1} \otimes I_{N+1}^{\otimes m-|w|}\right) S^{|w|}
$$

(in the case $|w|=m$, the last term in the tensor product above disappears, and $\left.S^{|w|}=I_{n}\right)$, or, on the basis vectors,

$Z(\lambda)^{w}\left(e_{i_{1}} \otimes \cdots \otimes e_{i_{m}}\right)=\lambda^{w}\left(E_{j_{1}+1,1} e_{i_{m-|w|+1}} \otimes \cdots \otimes E_{j_{|w|}+1,1} e_{i_{m}} \otimes e_{i_{1}} \otimes \cdots \otimes e_{i_{m-|w|}}\right)$.

Let us remark that since $\lambda_{k}, k=1, \ldots, N$, are scalars, and thus commute, we may rewrite $\lambda^{w}$ as $\lambda^{t(w)}$, where $t: \mathcal{F}_{N} \rightarrow \mathbb{Z}_{+}^{N}$ is the abelianization map: $t(w)=\left(t_{1}(w), \ldots, t_{N}(w)\right)$ with the non-negative integer number $t_{k}(w)$ equal to the numbers of times that the letter $g_{k}$ appears in the word $w$. Thus, we have

$$
\lambda^{w}=\lambda^{t(w)}=\lambda_{1}^{t_{1}(w)} \cdots \lambda_{N}^{t_{N}(w)}
$$

For $w=g_{j_{1}} \cdots g_{j_{|w|}} \in \mathcal{F}_{N}:|w|=m+1$, one has

$$
\begin{aligned}
Z(\lambda)^{w} & =\lambda^{t(w)}\left(E_{j_{1}+1,1} \otimes I_{N+1}^{\otimes m-1}\right) S\left(E_{j_{2}+1,1} \otimes \cdots \otimes E_{j_{m+1}, 1}\right) \\
& =\lambda^{t(w)}\left(E_{j_{1}+1,1} E_{j_{m+1}, 1} \otimes E_{j_{2}+1,1} \otimes \cdots \otimes E_{j_{m}, 1}\right) S=0
\end{aligned}
$$

since $E_{j+1,1} E_{k+1,1}=0$ for any $j, k \in\{1, \ldots, N\}$. Thus, $Z(\lambda) \in \operatorname{Nilp}_{N}(n, m+1)$, and by the assumption,

$$
\begin{aligned}
P\left(\lambda, \lambda^{\prime}\right) & =K\left(Z(\lambda), Z\left(\lambda^{\prime}\right)\right)=\sum_{w, w^{\prime} \in \mathcal{F}_{N}: 0 \leq|w|,\left|w^{\prime}\right| \leq m} K_{w, w^{\prime}} \otimes Z(\lambda)^{w} Z\left(\lambda^{\prime}\right)^{* w^{\prime \top}} \\
& =\sum_{t, t^{\prime} \in \mathbb{Z}_{+}^{N}: 0 \leq|t|,\left|t^{\prime}\right| \leq m}\left(\sum_{w, w^{\prime} \in \mathcal{F}_{N}: t(w)=t, t\left(w^{\prime}\right)=t^{\prime}} K_{w, w^{\prime}} \otimes Z^{w} Z^{* w^{\prime \top}}\right) \lambda^{t}\left(\overline{\lambda^{\prime}}\right)^{t^{\prime}}
\end{aligned}
$$

is a polynomial $\mathcal{L}\left(\mathcal{E} \otimes \mathbb{C}^{N}\right)$-valued function which is a positive kernel on $\mathbb{C}^{N}$. By Lemma1, the coefficients of this kernel serve as the operator blocks $P_{t, t^{\prime}} \in \mathcal{L}\left(\mathcal{E} \otimes \mathbb{C}^{n}\right)$ of the matrix $M_{P}=\left(P_{t, t^{\prime}}\right)_{0 \leq|t|,\left|t^{\prime}\right| \leq m}$ (for some order on $\mathbb{Z}_{+}^{N}$ ) which represents a 
positive semidefinite operator on the Hilbert space $\left(\mathcal{E} \otimes \mathbb{C}^{n}\right)^{\frac{(m+N) !}{m ! N !}}$. Therefore, for arbitrary vectors

$$
u_{t}=\sum_{1 \leq i_{1}, \ldots, i_{m} \leq N+1} u_{i_{1}, \ldots, i_{m}}^{(t)} \otimes e_{i_{1}} \otimes \cdots \otimes e_{i_{m}} \in \mathcal{E} \otimes \mathbb{C}^{n} \cong \mathcal{E} \otimes\left(\mathbb{C}^{N+1}\right)^{\otimes m}
$$

where $t \in \mathbb{Z}_{+}^{N}: 0 \leq|t| \leq m$, we can write:

$$
\begin{aligned}
0 & \leq \sum_{t, t^{\prime} \in \mathbb{Z}_{+}^{N}: 0 \leq|t|,\left|t^{\prime}\right| \leq m}\left\langle P_{t, t^{\prime}} u_{t^{\prime}}, u_{t}\right\rangle_{\mathcal{E} \otimes \mathbb{C}^{n}} \\
& =\left(\sum_{|t|=\left|t^{\prime}\right|}+\sum_{|t|>\left|t^{\prime}\right|}+\sum_{|t|<\left|t^{\prime}\right|}\right)\left\langle P_{t, t^{\prime}} u_{t^{\prime}}, u_{t}\right\rangle_{\mathcal{E} \otimes \mathbb{C}^{n}}=A+B+C .
\end{aligned}
$$

Let us calculate each of the sums $A, B$ and $C$ separately:

$$
\begin{aligned}
& A=\sum_{|t|=\left|t^{\prime}\right|}\left\langle P_{t, t^{\prime}} u_{t^{\prime}}, u_{t}\right\rangle_{\mathcal{E} \otimes \mathbb{C}^{n}} \\
& =\sum_{|t|=\left|t^{\prime}\right|}\left\langle\left(\sum_{w, w^{\prime} \in \mathcal{F}_{N}:} \sum_{t(w)=t, t\left(w^{\prime}\right)=t^{\prime}} K_{w, w^{\prime}} \otimes Z^{w} Z^{* w^{\prime \top}}\right) u_{t^{\prime}}, u_{t}\right\rangle_{\mathcal{E} \otimes \mathbb{C}^{n}} \\
& =\sum_{|t|=\left|t^{\prime}\right|} \sum_{t(w)=t, t\left(w^{\prime}\right)=t^{\prime}}\left\langle K_{w, w^{\prime}} \otimes\left(E_{j_{1}+1,1} \otimes \cdots \otimes E_{j_{|w|}+1,1} \otimes I_{N+1}^{\otimes m-|w|}\right) S^{|w|}\right. \\
& \left.\times S^{*\left|w^{\prime}\right|}\left(E_{1, k_{1}+1} \otimes \cdots \otimes E_{1, k_{\left|w^{\prime}\right|}+1} \otimes I_{N+1}^{\otimes m-\left|w^{\prime}\right|}\right) u_{t^{\prime}}, u_{t}\right\rangle_{\mathcal{E} \otimes \mathbb{C}^{n}} \\
& =\sum_{|t|=\left|t^{\prime}\right|} \sum_{t(w)=t, t\left(w^{\prime}\right)=t^{\prime}}\left\langle K_{w, w^{\prime}} \otimes E_{j_{1}+1, k_{1}+1} \otimes \cdots \otimes E_{j_{|w|}+1, k_{\left|w^{\prime}\right|}+1} \otimes I_{N+1}^{\otimes m-|t|}\right. \\
& \times \sum_{1 \leq i_{1}, \ldots, i_{m} \leq N+1} u_{i_{1}, \ldots, i_{m}}^{\left(t^{\prime}\right)} \otimes e_{i_{1}} \otimes \cdots \otimes e_{i_{m}}, \\
& \left.\sum_{1 \leq s_{1}, \ldots, s_{m} \leq N+1} u_{s_{1}, \ldots, s_{m}}^{(t)} \otimes e_{s_{1}} \otimes \cdots \otimes e_{s_{m}}\right\rangle_{\mathcal{E} \otimes \mathbb{C}^{n}} \\
& =\sum_{|t|=\left|t^{\prime}\right|} \sum_{t(w)=t, t\left(w^{\prime}\right)=t^{\prime}} \sum_{1 \leq i_{\left|t^{\prime}\right|+1}, \ldots, i_{m} \leq N+1}\left\langle K_{w, w^{\prime}} u_{k_{1}+1, \ldots, k_{\left|w^{\prime}\right|}+1, i_{\left|t^{\prime}\right|+1}, \ldots, i_{m}}^{\left(t^{\prime}\right)},\right. \\
& \left.u_{j_{1}+1, \ldots, j_{|w|}+1, i_{\left|t^{\prime}\right|+1}, \ldots, i_{m}}^{(t)}\right\rangle_{\mathcal{E}} \\
& B=\sum_{|t|>\left|t^{\prime}\right|}\left\langle P_{t, t^{\prime}} u_{t^{\prime}}, u_{t}\right\rangle_{\mathcal{E} \otimes \mathbb{C}^{n}} \\
& =\sum_{|t|>\left|t^{\prime}\right|}\left\langle\left(\sum_{w, w^{\prime} \in \mathcal{F}_{N}:} \sum_{t(w)=t, t\left(w^{\prime}\right)=t^{\prime}} K_{w, w^{\prime}} \otimes Z^{w} Z^{* w^{\prime \top}}\right) u_{t^{\prime}}, u_{t}\right\rangle_{\mathcal{E} \otimes \mathbb{C}^{n}}
\end{aligned}
$$




$$
\begin{aligned}
& =\sum_{|t|>\left|t^{\prime}\right|} \sum_{t(w)=t, t\left(w^{\prime}\right)=t^{\prime}}\left\langle K_{w, w^{\prime}} \otimes\left(E_{j_{1}+1,1} \otimes \cdots \otimes E_{j_{|w|}+1,1} \otimes I_{N+1}^{\otimes m-|w|}\right) S^{|w|}\right. \\
& \left.\times S^{*\left|w^{\prime}\right|}\left(E_{1, k_{1}+1} \otimes \cdots \otimes E_{1, k_{\left|w^{\prime}\right|}+1} \otimes I_{N+1}^{\otimes m-\left|w^{\prime}\right|}\right) u_{t^{\prime}}, u_{t}\right\rangle_{\mathcal{E} \otimes \mathbb{C}^{n}} \\
& =\sum_{|t|>\left|t^{\prime}\right|} \sum_{t(w)=t, t\left(w^{\prime}\right)=t^{\prime}}\left\langle K_{w, w^{\prime}} \otimes E_{j_{1}+1,1} \otimes \cdots \otimes E_{j_{|w|-\left|w^{\prime}\right|}+1,1}\right. \\
& \otimes E_{j_{|w|-\left|w^{\prime}\right|+1}+1, k_{1}+1} \otimes \cdots \otimes E_{j_{|w|}+1, k_{\left|w^{\prime}\right|}+1} \otimes I_{N+1}^{\otimes m-|t|} \\
& \times \sum_{1 \leq i_{1}, \ldots, i_{m} \leq N+1} u_{i_{1}, \ldots, i_{m}}^{\left(t^{\prime}\right)} \otimes e_{i_{m-|w|+\left|w^{\prime}\right|+1}} \otimes \cdots \otimes e_{i_{m}} \otimes e_{i_{1}} \otimes \cdots \otimes e_{i_{m-|w|+\left|w^{\prime}\right|}}, \\
& \left.\sum_{1 \leq s_{1}, \ldots, s_{m} \leq N+1} u_{s_{1}, \ldots, s_{m}}^{(t)} \otimes e_{s_{1}} \otimes \cdots \otimes e_{s_{m}}\right\rangle_{\mathcal{E} \otimes \mathbb{C}^{n}} \\
& =\sum_{|t|>\left|t^{\prime}\right|} \sum_{t(w)=t, t\left(w^{\prime}\right)=t^{\prime}} \sum_{1 \leq i_{\left|t^{\prime}\right|+1}, \ldots, i_{m-|t|+\left|t^{\prime}\right|} \leq N+1}\left\langle K_{w, w^{\prime}}\right. \\
& \left.\times u_{k_{1}+1, \ldots, k_{\left|w^{\prime}\right|}+1, i_{\left|t^{\prime}\right|+1}, \ldots, i_{m-|t|+\left|t^{\prime}\right|} \mid 1, \ldots, 1}^{\left(t^{\prime}\right)}, u_{j_{1}+1, \ldots, j_{|w|}+1, i_{\left|t^{\prime}\right|+1}, \ldots, i_{m-|t|+\left|t^{\prime}\right|}}^{(t)}\right\rangle_{\mathcal{E}} ; \\
& C=\sum_{|t|<\left|t^{\prime}\right|}\left\langle P_{t, t^{\prime}} u_{t^{\prime}}, u_{t}\right\rangle_{\mathcal{E} \otimes \mathbb{C}^{n}} \\
& =\sum_{|t|<\left|t^{\prime}\right|}\left\langle\left(\sum_{w, w^{\prime} \in \mathcal{F}_{N}:} \sum_{t(w)=t, t\left(w^{\prime}\right)=t^{\prime}} K_{w, w^{\prime}} \otimes Z^{w} Z^{* w^{\prime \top}}\right) u_{t^{\prime}}, u_{t}\right\rangle_{\mathcal{E} \otimes \mathbb{C}^{n}} \\
& =\sum_{|t|<\left|t^{\prime}\right|} \sum_{t(w)=t, t\left(w^{\prime}\right)=t^{\prime}}\left\langle K_{w, w^{\prime}} \otimes\left(E_{j_{1}+1,1} \otimes \cdots \otimes E_{j_{|w|}+1,1} \otimes I_{N+1}^{\otimes m-|w|}\right) S^{|w|}\right. \\
& \left.\times S^{*\left|w^{\prime}\right|}\left(E_{1, k_{1}+1} \otimes \cdots \otimes E_{1, k_{\left|w^{\prime}\right|}+1} \otimes I_{N+1}^{\otimes m-\left|w^{\prime}\right|}\right) u_{t^{\prime}}, u_{t}\right\rangle_{\mathcal{E} \otimes \mathbb{C}^{n}} \\
& =\sum_{|t|<\left|t^{\prime}\right|} \sum_{t(w)=t, t\left(w^{\prime}\right)=t^{\prime}}\left\langle K_{w, w^{\prime}} \otimes E_{j_{1}+1, k_{\left|w^{\prime}\right|-|w|+1}+1} \otimes \cdots \otimes E_{j_{|w|}+1, k_{\left|w^{\prime}\right|}+1}\right. \\
& \otimes I_{N+1}^{\otimes m-\left|t^{\prime}\right|} \otimes E_{1, k_{1}+1} \otimes \cdots \otimes E_{1, k_{\left|w^{\prime}\right|-|w|}+1} \\
& \times \sum_{1 \leq i_{1}, \ldots, i_{m} \leq N+1} u_{i_{1}, \ldots, i_{m}}^{\left(t^{\prime}\right)} \otimes e_{i_{\left|w^{\prime}\right|-|w|+1}} \otimes \cdots \otimes e_{i_{m}} \otimes e_{i_{1}} \otimes \cdots \otimes e_{i_{\left|w^{\prime}\right|-|w|}}, \\
& \left.\sum_{1 \leq s_{1}, \ldots, s_{m} \leq N+1} u_{s_{1}, \ldots, s_{m}}^{(t)} \otimes e_{s_{1}} \otimes \cdots \otimes e_{s_{m}}\right\rangle_{\mathcal{E} \otimes \mathbb{C}^{n}} \\
& =\sum_{|t|<\left|t^{\prime}\right|} \sum_{t(w)=t, t\left(w^{\prime}\right)=t^{\prime}} \sum_{1 \leq i_{\left|t^{\prime}\right|+1}, \ldots, i_{m} \leq N+1}\left\langle K_{w, w^{\prime}}\right. \\
& \left.\times u_{k_{1}+1, \ldots, k_{\left|w^{\prime}\right|}+1, i_{\left|t^{\prime}\right|+1}, \ldots, i_{m}}^{\left(t^{\prime}\right)}, u_{j_{1}+1, \ldots, j_{|w|}+1, i_{\left|t^{\prime}\right|+1}, \ldots, i_{m}, 1 \ldots, 1}^{(t)}\right\rangle_{\mathcal{E}} .
\end{aligned}
$$


Now, for $t \in \mathbb{Z}_{+}^{N}: 0 \leq|t| \leq m$, and $w=g_{j_{1}} \cdots g_{j_{|w|}} \in \mathcal{F}_{N}: t(w)=t$, set

$$
u_{j_{1}+1, \ldots, j_{|w|}+1, i_{|t|+1}, \ldots, i_{m}}^{(t)}=0 \text { for }\left(i_{|t|+1}, \ldots, i_{m}\right) \neq(1, \ldots, 1),
$$

and

$$
u_{j_{1}+1, \ldots, j_{|w|}+1,1, \ldots, 1}^{(t)}=u_{j_{1}+1, \ldots, j_{|w|}+1,1, \ldots, 1}^{(t(w))}=h_{w} \in \mathcal{E} .
$$

Then the inequality $A+B+C \geq 0$ turns into

$$
\sum_{w, w^{\prime} \in \mathcal{F}_{N}: 0 \leq|w|,\left|w^{\prime}\right| \leq m}\left\langle K_{w, w^{\prime}} h_{w^{\prime}}, h_{w}\right\rangle_{\mathcal{E}} \geq 0
$$

Since the integer $m>1$ and the vectors $h_{w} \in \mathcal{E}$ are chosen arbitrarily, this means that $K\left(z, z^{\prime}\right)$ is a positive non-commutative kernel (for the cases $m=0$ and $m=1$ the inequality above now follows automatically).

\section{Proof of Theorem 2}

For the proof of Theorem 2 we need the following lemma. (For a result of a similar nature in a commutative setting, see [5, Proposition 2.4].)

Lemma 2. An arbitrary convergent positive non-commutative kernel $K\left(z, z^{\prime}\right) \in$ $\mathcal{L}(\mathcal{E})\left\langle\left\langle z, z^{\prime}\right\rangle\right\rangle$ admits a factorization $K\left(z, z^{\prime}\right)=H(z) H\left(z^{\prime}\right)^{*}$ with a convergent formal power series $H(z) \in \mathcal{L}(\mathcal{H}, \mathcal{E})\left\langle\left\langle z, z^{\prime}\right\rangle\right\rangle$, for an auxiliary Hilbert space $\mathcal{H}$. (A formal power series $H(z) \in \mathcal{L}(\mathcal{H}, \mathcal{E})\left\langle\left\langle z, z^{\prime}\right\rangle\right\rangle$ is called convergent if for every $n \in \mathbb{N}$ there exists a connected open neighbourhood $V_{n}$ of 0 in $\left(\mathbb{C}^{n \times n}\right)^{N}$ such that the series $H(Z)$ as in (4) converges uniformly on compact subsets of $V_{n}$ in the norm of the Banach space $\mathcal{L}\left(\mathcal{H} \otimes \mathbb{C}^{n}, \mathcal{E} \otimes \mathbb{C}^{n}\right)$.)

Proof. Let $\left(\lambda^{\prime}, \lambda^{\prime \prime}\right) \in U_{1} \times U_{1}$, where $U_{n}$ is a neighbourhood of 0 in $\left(\mathbb{C}^{n \times n}\right)^{N}$ associated with $K\left(z, z^{\prime}\right)$ as in the definition of a convergent non-commutative formal power series which was given in Section 1. Then the series $K\left(\lambda^{\prime}, \lambda^{\prime \prime}\right)=$ $\sum_{w \in \mathcal{F}_{N}} K_{w, w^{\prime}} \lambda^{t(w)} \lambda^{\prime \prime t\left(w^{\prime}\right)}$ converges in the operator norm, and hence there exists a constant $M>0$ such that $\left\|K_{w, w^{\prime}}\right\| \rho^{\prime t(w)} \rho^{\prime \prime t\left(w^{\prime}\right)} \leq M$ for all $w, w^{\prime} \in \mathcal{F}_{N}$, where $\rho^{\prime}=\left(\left|\lambda_{1}^{\prime}\right|, \ldots,\left|\lambda_{N}^{\prime}\right|\right), \rho^{\prime \prime}=\left(\left|\lambda_{1}^{\prime \prime}\right|, \ldots,\left|\lambda_{N}^{\prime \prime}\right|\right)$, and for a $w \in \mathcal{F}_{N}$ the $N$-tuple of integers $t(w)$ is defined in Section 2, Set $\rho_{k}=\min \left(\left|\lambda_{k}^{\prime}\right|,\left|\lambda_{k}^{\prime \prime}\right|\right), k=1, \ldots, N$, and $\rho=\left(\rho_{1}, \ldots, \rho_{N}\right)$. Since $K\left(z, z^{\prime}\right)$ is a non-commutative positive kernel, it admits a factorization $K\left(z, z^{\prime}\right)=H(z) H\left(z^{\prime}\right)^{*}$ with a formal power series $H(z) \in$ $\mathcal{L}(\mathcal{H}, \mathcal{E})\left\langle\left\langle z, z^{\prime}\right\rangle\right\rangle$, for an auxiliary Hilbert space $\mathcal{H}$. This means that $K_{w, w^{\prime}}=H_{w} H_{w^{\prime}}^{*}$ for every $w, w^{\prime} \in \mathcal{F}_{N}$. Therefore we have

$$
\left\|K_{w, w}\right\| \rho^{2 t(w)}=\left\|H_{w} H_{w}^{*}\right\| \rho^{2 t(w)}=\left\|H_{w}\right\|^{2} \rho^{2 t(w)} \leq M, \quad w \in \mathcal{F}_{N} .
$$

We will now use an argument similar to the one used in the proof of Abel's lemma in the setting of several complex variables (see, e.g., [10). Denote by $V_{n}$ the open neighbourhood of 0 in $\left(\mathbb{C}^{n \times n}\right)^{N}$ defined by the inequality $\sum_{k=1}^{N} \rho_{k}^{-1}\left\|Z_{k}\right\|<1$. Then 
for $Z \in V_{n}$ we have

$$
\begin{aligned}
& \sum_{w \in \mathcal{F}_{N}}\left\|H_{w} \otimes Z^{w}\right\|=\sum_{w \in \mathcal{F}_{N}}\left\|H_{w}\right\| \cdot\left\|Z^{w}\right\| \leq \sum_{w \in \mathcal{F}_{N}}\left\|H_{w}\right\| \prod_{k=1}^{N}\left\|Z_{k}\right\|^{t_{k}(w)} \\
& \leq \sqrt{M} \sum_{w \in \mathcal{F}_{N}} \prod_{k=1}^{N}\left(\rho_{k}^{-1}\left\|Z_{k}\right\|\right)^{t_{k}(w)}=\sqrt{M} \sum_{m=0}^{\infty}\left(\sum_{k=1}^{N} \rho_{k}^{-1}\left\|Z_{k}\right\|\right)^{m} \\
&=\sqrt{M}\left(1-\sum_{k=1}^{N} \rho_{k}^{-1}\left\|Z_{k}\right\|\right)^{-1}<\infty
\end{aligned}
$$

Therefore for every $n \in \mathbb{N}$ the power series $H(Z)=\sum_{w \in \mathcal{F}_{N}} H_{w} \otimes Z^{w}$ converges absolutely and uniformly on compact subsets of $V_{n}$ in the norm of $\mathcal{L}\left(\mathcal{H} \otimes \mathbb{C}^{n}, \mathcal{E} \otimes \mathbb{C}^{n}\right)$, i.e., $H(z)$ is a convergent formal power series.

Remark 1. It is clear from the proof of Lemma2 that the assumption of convergence of the series $K\left(\lambda^{\prime}, \lambda^{\prime \prime}\right)$ at only one point $\left(\lambda^{\prime}, \lambda^{\prime \prime}\right) \in \mathbb{C}^{N} \times \mathbb{C}^{N}$ is already sufficient for the conclusion of this lemma. Moreover, this guarantees the existence of neighbourhoods $V_{n}(n \in \mathbb{N})$ "of the same size", i.e., with the same bounds on the norms of $Z_{k}, k=1, \ldots, N$, such that the series $H(Z)$ converges uniformly and absolutely on compact subsets of $V_{n}$.

Proof of Theorem 2, Assume that the convergent formal power series $K\left(z, z^{\prime}\right) \in$ $\mathcal{L}(\mathcal{E})\left\langle\left\langle z, z^{\prime}\right\rangle\right\rangle$ (with respect to a set of neighbourhoods $U_{n}$ of 0 in $\left(\mathbb{C}^{n \times n}\right)^{N}, n \in$ $\mathbb{N})$ is a positive non-commutative kernel. Then by Lemma $2 K\left(z, z^{\prime}\right)$ admits a factorization $K\left(z, z^{\prime}\right)=H(z) H\left(z^{\prime}\right)^{*}$ for some convergent formal power series $H(z)$ as in (21), i.e., for every $n \in \mathbb{N}$ there exists a neighbourhood $V_{n}$ of 0 in $\left(\mathbb{C}^{n \times n}\right)^{N}$, such that the series $H(Z)=\sum_{w \in \mathcal{F}_{N}} H_{w} \otimes Z^{w}$ converges uniformly on compact subsets of $V_{n}$ (in the norm of the Banach space $\mathcal{L}\left(\mathcal{H} \otimes \mathbb{C}^{n}, \mathcal{E} \otimes \mathbb{C}^{n}\right)$ ). Since we can replace $V_{n}$ by $V_{n} \cap U_{n}$ for all $n \in \mathbb{N}$, without loss of generality we may assume that $V_{n} \subset U_{n}, n \in \mathbb{N}$. In this case $K\left(Z, Z^{\prime}\right)$ is a positive $\mathcal{L}\left(\mathcal{E} \otimes \mathbb{C}^{n}\right)$-valued kernel on $U_{n}$. (We first see that $K\left(Z, Z^{\prime}\right)=H(Z) H\left(Z^{\prime}\right)^{*}$ is a positive kernel on $V_{n}$, then observe that due to uniform convergence of the series for $K\left(Z, Z^{\prime}\right)$ on compact subsets of $U_{n}$ the function $K\left(Z, Z^{\prime}\right)$ is sesquianalytic, and then use the fact that for sesquianalytic kernels the positivity property propagates to an arbitrarily large region on which the kernel is sesquianalytic; see [1, Theorem 1.1.4] in the one-dimensional case the general case is completely analogous.)

Conversely, if $K\left(z, z^{\prime}\right) \in \mathcal{L}(\mathcal{E})\left\langle\left\langle z, z^{\prime}\right\rangle\right\rangle$ is a convergent formal power series, and if $K\left(Z, Z^{\prime}\right)$ is a positive $\mathcal{L}\left(\mathcal{E} \otimes \mathbb{C}^{n}\right)$-valued kernel on $U_{n}$ for every $n \in \mathbb{N}$, then $K\left(z, z^{\prime}\right)$ is a positive non-commutative kernel. The proof is the same as in Section2. except that the polynomial $P\left(\lambda, \lambda^{\prime}\right)$ is guaranteed to be a positive kernel only on a neighbourhood of 0 in $\mathbb{C}^{N}$. It is clear from the proof of Lemma 1 that this still implies positive semidefiniteness of the block operator matrix $M_{P}$ (alternatively, one can use the propagation property of positivity for sesquianalytic kernels, mentioned above, to first show that $P\left(\lambda, \lambda^{\prime}\right)$ is a positive kernel on all of $\left.\mathbb{C}^{N}\right)$.

\section{Proof of Theorems 3 and 4}

Proof of Theorem 3, As we already said in Section 1, one direction of this theorem is obvious, and thus only the other direction is left to prove. Assume that a formal power series $K\left(z, z^{\prime}\right) \in \mathcal{L}(\mathcal{E})\left\langle\left\langle z, z^{\prime}\right\rangle\right\rangle$ as in (11) is such that for every $n \in \mathbb{N}$ and 
$Z \in \operatorname{Nilp}_{N}(n)$ the operator $K(Z, Z)$ as in (5) is positive semidefinite. Fix $m \in \mathbb{N}$, and for arbitrary $s>0$ denote by $\mathcal{H}_{s}$ the Hilbert space with orthogonal basis equal to the set $\mathcal{F}_{N}^{(m)}$ of words $w \in \mathcal{F}_{N}$ of length at most $m$, normalized by $\langle w, w\rangle=s^{-|w|}$. Let $S^{*}$ denote the backward shift $N$-tuple on $\mathcal{H}_{s}$ defined as $S_{j}^{*} w=v$ if $w=g_{j} v$, and $S_{j}^{*} w=0$ otherwise. Clearly, $S \in \operatorname{Nilp}_{N}\left(\sum_{j=0}^{m} N^{j}, m+1\right)$. Given vectors $h_{\alpha} \in \mathcal{E}\left(\alpha \in \mathcal{F}_{N}^{(m)}\right)$, compute

$$
\begin{aligned}
\left\langle K(S, S) \sum_{\alpha \in \mathcal{F}_{N}^{(m)}} h_{\alpha} \otimes \alpha, \sum_{\beta \in \mathcal{F}_{N}^{(m)}} h_{\beta} \otimes \beta\right\rangle_{\mathcal{E} \otimes \mathcal{H}_{s}} \\
=\left\langle\left(\sum_{w, w^{\prime} \in \mathcal{F}_{N}^{(m)}} K_{w, w^{\prime}} \otimes S^{w} S^{* w^{\prime T}}\right) \sum_{\alpha \in \mathcal{F}_{N}^{(m)}} h_{\alpha} \otimes \alpha, \sum_{\beta \in \mathcal{F}_{N}^{(m)}} h_{\beta} \otimes \beta\right\rangle \\
=\sum_{\mathcal{E} \otimes \mathcal{H}_{s}} \sum_{\alpha, w^{\prime} \in \mathcal{F}_{N}^{(m)}}\left\langle K_{w, w^{\prime}} h_{\alpha}, h_{\beta}\right\rangle_{\mathcal{E}}\left\langle S^{* w^{\prime} T} \alpha, S^{* w^{T}} \beta\right\rangle_{\mathcal{H}_{s}} \\
=\sum_{\gamma \in \mathcal{F}_{N}^{(m)}} \sum_{w, w^{\prime} \in \mathcal{F}_{N}^{(m-|\gamma|)}}\left\langle K_{w, w^{\prime}} h_{w^{\prime} \gamma}, h_{w \gamma}\right\rangle_{\mathcal{E}}\langle\gamma, \gamma\rangle_{\mathcal{H}_{s}} \\
=\sum_{\gamma \in \mathcal{F}_{N}^{(m)}} \sum_{w, w^{\prime} \in \mathcal{F}_{N}^{(m-|\gamma|)}}\left\langle K_{w, w^{\prime}} h_{w^{\prime} \gamma}, h_{w \gamma}\right\rangle_{\mathcal{E}} s^{-|\gamma|} .
\end{aligned}
$$

By hypothesis, $K(S, S)$ is a positive semidefinite operator on $\mathcal{H}_{s}$ for each $s>0$. Letting $s$ tend to infinity gives

$$
\sum_{w, w^{\prime} \in \mathcal{F}_{N}^{(m)}}\left\langle K_{w, w^{\prime}} h_{w^{\prime}}, h_{w}\right\rangle_{\mathcal{E}} \geq 0
$$

which, by virtue of arbitrariness of $m \in \mathbb{N}$ and of vectors $h_{w} \in \mathcal{E}\left(w \in \mathcal{F}_{N}^{(m)}\right)$, means that $K\left(z, z^{\prime}\right)$ is a positive non-commutative kernel.

Proof of Theorem 4. The statement follows from Theorem 3, since in this case $K\left(z, z^{\prime}\right) \in \mathcal{L}(\mathcal{E})\left\langle z, z^{\prime}\right\rangle$ is a positive non-commutative kernel. The estimate for $\operatorname{dim}(\mathcal{H})$ is easily obtained from the fact that the matrix $\left(K_{w, w^{\prime}}\right)_{|w| \leq m,\left|w^{\prime}\right| \leq m}$ with blocks from $\mathcal{L}(\mathcal{E})$ is of size $\left(\sum_{j=0}^{m} N^{j}\right) \times\left(\sum_{j=0}^{m} N^{j}\right)$ and positive semidefinite. The last statement of the theorem (about the size and the joint rank of nilpotency of the test matrices) follows from the proof of Theorem 3 .

\section{REFERENCES}

[1] D. Alpay, A. Dijksma, J. Rovnyak, and H. de Snoo, Schur functions, operator colligations, and reproducing kernel Pontryagin spaces, Operator Theory: Adv. Appl. 96, Birkhäuser Verlag, Basel, 1997. MR1465432 (2000a:47024)

[2] D. Alpay and D.S. Kalyuzhnyı̆-Verbovetzkĭ̌, On the intersection of null spaces for matrix substitutions in a non-commutative rational formal power series, C. R. Math. Acad. Sci. Paris 339 (2004), no. 8, 533-538.

[3] N. Aronszajn, Theory of reproducing kernels, Trans. Amer. Math. Soc. 68 (1950), 337-404. MR0051437 (14:479c)

[4] J.A. Ball, G. Groenewald, and T. Malakorn, Conservative structured noncommutative multidimensional linear systems, Multidimens. Syst. Signal Process., to appear. 
[5] J.A. Ball and V. Vinnikov, Formal reproducing kernel Hilbert spaces: The commutative and noncommutative settings, in Reproducing kernel spaces and applications, 77-134, Operator Theory Adv. Appl. 143, Birkhäuser Verlag, Basel, 2003. MR2019348(2005a:46058)

[6] J.W. Helton, "Positive" noncommutative polynomials are sums of squares, Ann. of Math. (2) 156 (2002). MR.1933721 (2003k:12002)

[7] J.W. Helton, S. McCullough, and M. Putinar, A non-commutative Positivstellensatz on isometries, J. Reine Angew. Math. 568 (2004), 71-80. MR2034923 (2004m:47026)

[8] S. McCullough, Factorization of operator-valued polynomials in several non-commuting variables, Linear Algebra Appl. 326 (2001), no. 1-3, 193-203. MR.1815959 (2002f:47035)

[9] S. Saitoh, Theory of Reproducing Kernels and its Applications, Pitman Res. Notes Math. 189, Longman Scientific and Technical, Harlow, 1988. MR0983117 (90f:46045)

[10] B.V. Shabat, Introduction to Complex Analysis. Part II, Translations of Mathematical Monographs 110, Functions of several variables, Translated from the third (1985) Russian edition by J. S. Joel, American Mathematical Society, Providence, RI, 1992. MR.1192135 (93g:32001)

Department of Mathematics, Ben-Gurion University of the Negev, Beer Sheva, IsRAEL 84105

E-mail address: dmitryk@math.bgu.ac.il

Department of Mathematics, Ben-Gurion University of the Negev, Beer Sheva, IsRAEL 84105

E-mail address: vinnikov@math.bgu.ac.il 